\title{
Vibration Analysis of Timoshenko Beams Traversed by Moving Loads
}

Yih-Hwang Lin

Department of Mechanical and Marine Engineering National Taiwan Ocean University Keelung 20224, Taiwan, Republic of China

Follow this and additional works at: https://jmstt.ntou.edu.tw/journal

Part of the Mechanical Engineering Commons

\section{Recommended Citation}

Lin, Yih-Hwang (1994) "Vibration Analysis of Timoshenko Beams Traversed by Moving Loads," Journal of Marine Science and Technology. Vol. 2: Iss. 1, Article 4.

DOI: $10.51400 / 2709-6998.2485$

Available at: https://jmstt.ntou.edu.tw/journal/vol2/iss1/4

This Research Article is brought to you for free and open access by Journal of Marine Science and Technology. It has been accepted for inclusion in Journal of Marine Science and Technology by an authorized editor of Journal of Marine Science and Technology. 


\section{Vibration Analysis of Timoshenko Beams Traversed by Moving Loads}

Acknowledgements

The author is grateful to the National Science Council (NSC), Taiwan, ROC, for the financial support under the contract NSC 81-0403-E-019-514. 


\title{
VIBRATION ANALYSIS OF TIMOSHENKO BEAMS TRAVERSED BY MOVING LOADS
}

\author{
Yih-Hwang Lin \\ Department of Mechanical and Marine Engineering \\ National Taiwan Ocean University \\ Keelung 20224, Taiwan, Republic of China
}

Key words: Timoshenko beams, moving loads, mode superposition.

\begin{abstract}
The purpose of this research is to apply the finite element method for dynamic analysis of Timoshenko beams traversed by moving loads. When an elastic structure is subjected to moving loads, the induced displacements and stresses are much higher than those of static loading conditions. Due to the prevalence of high speed machinery and the use of light and flexible structures to reduce the material cost, structural weight, and space requirement, dynamic analysis of such systems becomes crucial to ensure structural safety and required accuracy. In this study, the shear effect and rotary inertia were included for the analysis of short, sturdy beams and structures with higher modes being excited. This research will be applicable for dynamic analysis of bridges and rail roads subjected to moving vehicle loads, high speed machining, and structures supporting high speed moving objects, etc.
\end{abstract}

\section{INTRODUCTION}

The analysis of moving loads on an elastic structure has been a topic of interest for well over a century. Interest in this problem originated in civil engineering for the design of railroad bridges and highway structures. The problem arose from the observations that as a structure is subjected to moving loads, the dynamic deflection, as well as stresses, can be significantly higher than those for static loads. Most of the previous analysis work in this area were directed at the dynamic behavior of a simple structure, such as a simply supported beam, subjected to a simple loading, e.g. a concentrated force [1-3]. Different types of loading conditions for simple structures are well documented in [4]. The case of a rotating beam subjected to deflection dependent moving forces was studied by Katz et al. [5]. It was found that instability may occur for structures excited by a sequence of moving loads. The first attempt to solve the moving load problem with an undamped two-axle moving system was made by Wen [6]. The governing equations were derived using Lagrange's equation. The support beam was simply supported and the moving system was assumed to travel at a constant velocity. Discussion of the experimental verification of the theory developed in Ref. [6] was pre- sented in $[7,8]$. To handle more complex situations many researchers have applied the finite element method to study the dynamic behavior of structures under moving loads [9-11]. Galerkin's method was used to derive the governing equations. A review on the use of finite element method for moving load problem is given in [12]. Dynamic analysis and experimental model verification for a general moving load problem concerning a complex industrial high speed drilling machine were reported $[13,14]$. The analyses were valid for long, slender beam structures. For more accurate analysis, the effects of rotary inertia and shearing deformations need to be considered for short, sturdy beam structures or systems with higher modes being excited. Some research work has also been conducted regarding Timoshenko beams subjected to a moving force [15-18]. However, the analyses were performed for beams with simple boundary conditions so that analytical derivation was possible using simple functions. For more complex systems with general boundary conditions, finite element analysis technique is ideal for engineering analysis.

\section{TIMOSHENKO BEAM DYNAMICS}

For short, sturdy beams, the shear effect can not be 
neglected as in conventional analysis using BernoulliEuler's beam theory. The situation occurs when the the cross section of the beam is relatively large in comparison with the beam span. Note that although the correction for shear effect may yield results only a few percent more accurate in frequency prediction than those from classical beam theory for a moderately thick beam, the accuracy improvement may be quite profound when performing dynamic response analysis.

A straight forward energy minimization approach was reported in Ref. [19] for static analysis, which yields correct finite element characteristics without using additional finite element nodal degrees of freedoms when transverse shear effect is included. A traditional cubic polynomial can still be used to describe the transverse displacement. Constant shear strain within an element cross-section is assumed and is described as:

$$
\gamma=\frac{\partial w}{\partial x}-\theta
$$

where ' $w$ ' denotes transverse displacement of the beam, ' $\theta$ ' the cross section rotation, and ' $\gamma$ the shear strain.

The following shape functions for both the transverse displacement and cross-section rotation can be obtained from a straightforward derivation:

$$
\begin{aligned}
& w=[N]\{d\}_{e}, \quad \theta=[\bar{N}]\{d\}_{e} \\
& N_{1}=1-\frac{1}{a\left(a^{2}+12 g\right)}\left(12 g x+3 a x^{2}-2 x^{3}\right) \\
& N_{2}=\frac{1}{a\left(a^{2}+12 g\right)}\left[\left(a^{2}+6 g\right) a x-\left(2 a^{2}+6 g\right) x^{2}+a x^{3}\right] \\
& N_{3}=\frac{1}{a\left(a^{2}+12 g\right)}\left(12 g x+3 a x^{2}-2 x^{3}\right)
\end{aligned}
$$$$
N_{4}=\frac{1}{a\left(a^{2}+12 g\right)}\left[-6 g a x+\left(6 g-a^{2}\right) x^{2}+a x^{3}\right]
$$

and

$$
\begin{aligned}
& \bar{N}_{1}=\frac{1}{a\left(a^{2}+12 g\right)}\left(6 x^{2}-6 a x\right) \\
& \bar{N}_{2}=\frac{1}{a\left(a^{2}+12 g\right)}\left[a^{3}+12 g a-\left(4 a^{2}+12 g\right) x+3 a x^{2}\right] \\
& \bar{N}_{3}=\frac{1}{a\left(a^{2}+12 g\right)}\left(6 a x-6 x^{2}\right)
\end{aligned}
$$

$$
\bar{N}_{4}=\frac{1}{a\left(a^{2}+12 g\right)}\left[3 a x^{2}-\left(2 a^{2}-12 g\right) x\right]
$$

where

$$
g \equiv \frac{E I}{\mathrm{k} G A}
$$

and $[N]$ and $[\bar{N}]$ denote $1 \times 4$ row vectors representing shape functions, $\{d\}_{e}$ the element nodal degrees of freedom vector including transverse displacements and rotations, ' $a$ ' the beam element length, $E I$ the bending rigidity, $\mathrm{k}$ the shear coefficient, $\mathrm{G}$ the shear modulus, $\mathrm{A}$ the cross-section of the beam element, and ' $x$ ' the coordinate along the longitudinal direction of the beam element.

The strain energy including the shear effect for a beam element of length, $a$, can be described as:

$$
U_{e}=\frac{1}{2} \int_{0}^{a} E I\left(\frac{\partial \theta}{\partial x}\right)^{2} d x+\frac{1}{2} \int_{0}^{a} \mathrm{k} G A \gamma^{2} d x
$$

The stiffness matrix can be obtained directly from the description of strain energy. Substitution of Eqs. (1) and (2) into Eq. (6), we get

$$
\begin{aligned}
U_{e}= & \frac{1}{2}\{d\}_{e}^{T}\left(\int_{0}^{a} E I[\bar{N}]^{T}[\bar{N}] d x\right)\{d\}_{e} \\
& +\frac{1}{2}\{d\}_{e}^{T}\left(\int_{0}^{a} \operatorname{kGA}\left(\left[N_{x}\right]^{T}-[\bar{N}]^{T}\right)\right. \\
& \left.\left(\left[N_{x}\right]-[\bar{N}]\right) d x\right)\{d\}_{e} \\
= & \frac{1}{2}\{d\}_{e}^{T}\left[k_{b}\right]_{e}\{d\}_{e}+\frac{1}{2}\{d\}_{e}^{T}\left[k_{s}\right]_{e}\{d\}_{e}
\end{aligned}
$$

Where

$$
\left[k_{b}\right]_{e}=\int_{0}^{a} E I[\bar{N}]^{T}[\bar{N}] d x
$$

representing the bending effect and

$$
\left[k_{s}\right]_{e}=\int_{0}^{a} \operatorname{kGA}\left(\left[N_{x}\right]^{T}-[\bar{N}]^{T}\right)\left(\left[N_{x}\right]-[\bar{N}]\right) d x
$$

describing the shear effect, thus

$$
[k]_{e}=\left[k_{b}\right]_{e}+\left[k_{s}\right]_{e}
$$

and can be written as: 


$$
[k]_{e}=\frac{12}{\left(a^{2}+12 g\right)} \frac{E I}{a}\left[\begin{array}{cccc}
1 & & & \text { sym } \\
\frac{a}{2} & \frac{a^{2}}{3}+g & & \\
-1 & -\frac{a}{2} & 1 & \\
\frac{a}{2} & \frac{a^{2}}{6}-g & -\frac{a}{2} & \frac{a^{2}}{3}+g
\end{array}\right]
$$

which is the element stiffness matrix including the traditional bending effect with the addition of shear effect. Note that if ' $g$ ' is zero, which represents an infinite shear rigidity, the matrix reduces to the classical element stiffness matrix using Bernoulli-Euler's beam theory.

Eq. (9) was available in [19], in which only the static analysis of short beams considering the effect of shearing deformations was presented. For dynamic analysis of a short, sturdy beam traversed by moving loads considering both the effects of shearing deformations and rotary inertia, the mass matrix including these effects needs to be determined in addition to the previous development. Using the shape functions described previously, the kinetic energy of the beam can be written as:

$$
T_{e}=\frac{1}{2} \int_{0}^{a} \rho A\left(\frac{\partial w}{\partial t}\right)^{2} d x+\frac{1}{2} \int_{0}^{a} \rho I\left(\frac{\partial \theta}{\partial t}\right)^{2} d x
$$

substituting the shape functions and knowing that they are functions of $x$ only, we obtain

$$
\begin{aligned}
T_{e}= & \frac{1}{2}\{\dot{d}\}_{e}^{T}\left(\int_{0}^{a} \rho A[N]^{T}[N] d x\right)\{\dot{d}\}_{e}+\frac{1}{2}\{\dot{d}\}_{e}^{T} \\
& \left(\int_{0}^{a} \rho I[\bar{N}]^{T}[\bar{N}] d x\right)\{\dot{d}\}_{e} \\
= & \frac{1}{2}\{\dot{d}\}_{e}^{T}\left[m_{t}\right]_{e}\{\dot{d}\}_{e}+\frac{1}{2}\{\dot{d}\}_{e}^{T}\left[m_{r}\right]_{e}\{\dot{d}\}_{e}
\end{aligned}
$$

where $\left[m_{t}\right]_{e}$ represents the traditional mass matrix for transverse inertia effect, whereas $\left[m_{r}\right]_{e}$ describes the additional rotary inertia effect. These two matrices combined to form the element mass matrix to be shown subsequently.

Eq. (11) shows the correct description for considering both the transverse and rotary inertia. They are combined to form the mass matrix as:

$$
[m]_{e}=\left[m_{t}\right]_{e}+\left[m_{r}\right]_{e}
$$

After tedious algebra work performed by this author, the elements within the matrices are shown below:

$$
\begin{aligned}
{\left[m_{t}\right]_{e} } & =\int_{0}^{a}[N]^{T} \rho A[N] d x \\
& =\frac{\rho A a}{\left(\mathrm{a}^{2}+12 g\right)^{2}}\left[\begin{array}{llll}
t_{11} & & s y m \\
t_{21} & t_{22} & & \\
t_{31} & t_{32} & t_{33} & \\
t_{41} & t_{42} & t_{43} & t_{44}
\end{array}\right] \\
{\left[m_{r}\right]_{e} } & =\int_{0}^{a}[\bar{N}]^{T} \rho I[\bar{N}] d x \\
& =\frac{\rho l a}{\left(\mathrm{a}^{2}+12 g\right)^{2}}\left(\frac{r}{a}\right)^{2}\left[\begin{array}{llll}
r_{11} & & \\
r_{21} & r_{22} & & \\
r_{31} & r_{32} & r_{33} & \\
r_{41} & r_{42} & r_{43} & r_{44}
\end{array}\right]
\end{aligned}
$$

with

$$
\begin{aligned}
& t_{11}=\left(\frac{13}{35} a^{4}+\frac{42}{5} g a^{2}+48 g^{2}\right) \\
& t_{21}=\left(\frac{11}{210} a^{4}+\frac{11}{10} g a^{2}+6 g^{2}\right) a \\
& t_{22}=\left(\frac{1}{105} a^{4}+\frac{1}{5} g a^{2}+\frac{6}{5} g^{2}\right) a^{2} \\
& t_{31}=\left(\frac{9}{70} a^{4}+\frac{18}{5} g a^{2}+24 g^{2}\right) \\
& t_{32}=\left(\frac{13}{420} a^{4}+\frac{9}{10} g a^{2}+6 g^{2}\right) a \\
& t_{33}=t_{11} \\
& t_{41}=-t_{32} \\
& t_{42}=-\left(\frac{1}{140} a^{4}+\frac{1}{5} g a^{2}+\frac{6}{5} g^{2}\right) a^{2} \\
& t_{43}=-t_{21} \\
& t_{44}=t_{22}
\end{aligned}
$$

and

$$
\begin{aligned}
& r_{11}=\frac{6}{5} a^{4} \\
& r_{21}=\left(\frac{1}{10} a^{2}-6 g^{2}\right) a^{3} \\
& r_{22}=\left(\frac{2}{15} a^{4}+2 g a^{2}+48 g^{2}\right) a^{2} \\
& r_{31}=-r_{11} \\
& r_{32}=-r_{21} \\
& r_{33}=r_{11} \\
& r_{41}=r_{21}
\end{aligned}
$$




$$
\begin{aligned}
& r_{42}=\left(-\frac{1}{30} a^{4}-2 g a^{2}+24 g^{2}\right) a^{2} \\
& r_{43}=-r_{21} \\
& r_{44}=r_{22}
\end{aligned}
$$

The element mass and stiffness matrices have been derived to include the effects of shear and rotary inertia without using additional degrees of freedom at the node. This facilitates analysis for complex structural system without creating difficulties in treating the connection of dissimilar elements. Modal analysis or forced response can then be performed using this more accurate finite element formulation.

To examine the validity and characteristics of the finite element model developed in this paper, standard eigenvalue problem was solved. The comparison of normalized frequency parameters for a simply supported rectangular Timoshenko beam using the finite element formulation with those obtained using exact analysis [20] and Bernoulli-Euler's beam theory is tabulated in Table 1, with 32 elements being used. The slenderness ration $r / L$ is 0.015 , where ' $r$ ' is the radius of gyration of the cross section and ' $L$ ' the beam length. This is a relatively thin beam. The shear coefficient, $\mathrm{k}=0.85$, was used, which was derived by Cowper [21] by integrating the three-dimensional elasticity equations. Since a simply supported beam can exhibit two separate spectrums, that is, two distinct natural frequencies for each mode shape [22], the frequency type is denoted as low or high to indicate which spectrum the mode belongs to. As can be seen in Table 1, the finite element method yields very good results, whereas the results from Bernoulli-Euler beam theory have large errors, especially for higher modes.

The results for larger slenderness ratio for $\mathrm{r} / \mathrm{L}=$ 0.045 and 0.075 are tabulated in Tables 2 and 3, respectively. In Table 2, mode 9 was identified as shear mode, which is not shown from the exact [20] analysis. It was calculated using the formula given in [22]. This mode represents a rotation of the cross-section without transverse displacement as will be shown in the mode shape plots to be discussed later. The finite element formulation is able to predict this mode with sufficient accuracy. The tenth mode is shown to be the first high mode which depicts a mode with no nodal point between the beam supports. The Bernoulli-Euler's beam theory still predicts a mode with nine nodal points and is completely erroneous in describing the dynamic behavior of the beam system. In Table 3, the fifth mode is identified as the shear mode, whereas the first high mode shows up at the seventh mode. The error of frequency prediction from the Bernoulli-Euler's beam theory has been quite unacceptable for analysis of this relatively thick beam, whereas the finite element formulation still performs satisfactorily.

As just discussed previously, a simply supported beam exhibits two spectrums. Fig. 1 illustrates the mass normalized mode shape plots for the first ten modes for $\mathrm{r} / \mathrm{L}=0.015$. The beam is relatively thin and higher spectrum does not show up in the frequency range analyzed. Therefore, the mode shapes look similar to those obtained from classical analysis.

From Fig. 2, for $\mathrm{r} / \mathrm{L}=0.045$, the first 8 modes appear to be quite normal. However, as shown in Table 2 , the ninth mode is the shear mode and no transverse displacement is found in this mode. The tenth mode is the first mode in the higher spectrum and no nodal point within the beam span is present.

Fig. 3 depicts the first ten mode shapes for $r / L=$ 0.075 , a relatively thick beam. The shear oscillation appears at the fifth mode and shows no transverse displacement. Mode 7 is the first mode in the higher spectrum and has no nodal points between the beam span, where as mode 9 is the second mode in the higher spectrum and

Table 1. Comparison of frequency parameters $w_{n} \sqrt{\frac{\rho A L^{4}}{E I}}$ for a simply supported Timoshenko beam for $v=0.3, \mathrm{k}=0.85$,
and $\frac{r}{L}=0.015$.

\begin{tabular}{rcclc}
\hline Mode & $\begin{array}{c}\text { Freq. type } \\
\text { (low or high) }\end{array}$ & Exact [24] & FEM (\%Error) & B.-E(\% Error) \\
\hline 1 & 1 Low & $9.8255 \mathrm{e}+00$ & $9.8255 \mathrm{e}+00(0)$ & $9.8696 \mathrm{e}+00(0.45)$ \\
2 & 2 Low & $3.8790 \mathrm{e}+01$ & $3.8791 \mathrm{e}+01(0.00)$ & $3.9478 \mathrm{e}+01(1.78)$ \\
3 & 3 Low & $8.5474 \mathrm{e}+01$ & $8.5471 \mathrm{e}+01(0.02)$ & $8.8826 \mathrm{e}+01(3.92)$ \\
4 & 4 Low & $1.4785 \mathrm{e}+02$ & $1.4793 \mathrm{e}+02(0.06)$ & $1.5791 \mathrm{e}+02(6.80)$ \\
5 & 5 Low & $2.2361 \mathrm{e}+02$ & $2.2391 \mathrm{e}+02(0.14)$ & $2.4674 \mathrm{e}+02(10.34)$ \\
6 & 6 Low & $3.1049 \mathrm{e}+02$ & $3.1131 \mathrm{e}+02(0.26)$ & $3.5530 \mathrm{e}+02(14.44)$ \\
7 & 7 Low & $4.0640 \mathrm{e}+02$ & $4.0823 \mathrm{e}+02(0.45)$ & $4.8361 \mathrm{e}+02(19.00)$ \\
8 & 8 Low & $5.0956 \mathrm{e}+02$ & $5.1314 \mathrm{e}+02(0.71)$ & $6.3165 \mathrm{e}+02(23.96)$ \\
9 & 9 Low & $6.1847 \mathrm{e}+02$ & $6.2485 \mathrm{e}+02(1.03)$ & $7.9943 \mathrm{e}+02(29.26)$ \\
10 & 10 Low & $7.3191 \mathrm{e}+02$ & $7.4244 \mathrm{e}+02(1.44)$ & $9.8696 \mathrm{e}+02(34.85)$ \\
\hline
\end{tabular}


Table 2. Comparison of frequency parameters $w_{n} \sqrt{\frac{\rho A L^{4}}{E I}}$ for a simply supported Timoshenko beam for $v=0.3, k=0.85$, and $\frac{r}{L}=\mathbf{0 . 0 4 5}$.

\begin{tabular}{rcccc}
\hline Mode & $\begin{array}{c}\text { Freq. type } \\
\text { (low or high) }\end{array}$ & Exact [24] & FEM(\%Error) & B.-E(\% Error) \\
\hline 1 & 1 Low & $9.491 \mathrm{e}+00$ & $9.4973 \mathrm{e}+00(0.03)$ & $9.8696 \mathrm{e}+00(3.96)$ \\
2 & 2 Low & $3.4499 \mathrm{e}+01$ & $3.4508 \mathrm{e}+01(0.03)$ & $3.9478 \mathrm{e}+01(14.43)$ \\
3 & 3 Low & $6.8719 \mathrm{e}+01$ & $6.8797 \mathrm{e}+01(0.12)$ & $8.8826 \mathrm{e}+01(29.26)$ \\
4 & 4 Low & $1.0763 \mathrm{e}+02$ & $1.0792 \mathrm{e}+02(0.27)$ & $1.5791 \mathrm{e}+02(46.72)$ \\
5 & 5 Low & $1.4878 \mathrm{e}+02$ & $1.4956 \mathrm{e}+02(0.52)$ & $2.4674 \mathrm{e}+02(65.84)$ \\
6 & 6 Low & $1.9093 \mathrm{e}+02$ & $1.9258 \mathrm{e}+02(0.86)$ & $3.5530 \mathrm{e}+02(86.09)$ \\
7 & 7 Low & $2.3346 \mathrm{e}+02$ & $2.3649 \mathrm{e}+02(1.30)$ & $4.8361 \mathrm{e}+02(107.15)$ \\
8 & 8 Low & $2.7606 \mathrm{e}+02$ & $2.8109 \mathrm{e}+02(1.82)$ & $6.3165 \mathrm{e}+02(128.81)$ \\
9 & Shear mode & $2.8236 \mathrm{e}+02$ & $2.8421 \mathrm{e}+02(0.66)$ & $7.9943 \mathrm{e}+02(183.12)$ \\
10 & 10 Low & $2.9345 \mathrm{e}+02$ & $2.9559 \mathrm{e}+02(0.73)$ & $9.8696 \mathrm{e}+02(236.33)$ \\
\hline
\end{tabular}

Table 3. Comparison of frequency parameters $w_{n} \sqrt{\frac{\rho A L^{4}}{E I}}$ for a simply supported Timoshenko beam for $v=0.3, \mathrm{k}=0.85$, and $\frac{r}{L}=\mathbf{0 . 0 7 5}$.

\begin{tabular}{rcccc}
\hline Mode & $\begin{array}{c}\text { Freq. type } \\
\text { (low or high) }\end{array}$ & Exact [24] & EM (\%Error) & B.-E(\% Error) \\
\hline 1 & 1 Low & $8.9444 \mathrm{e}+00$ & $8.9449 \mathrm{e}+00(0.01)$ & $9.8696 \mathrm{e}+00(10.34)$ \\
2 & 2 Low & $2.9276 \mathrm{e}+01$ & $2.9293 \mathrm{e}+01(0.06)$ & $3.9478 \mathrm{e}+01(34.85)$ \\
3 & 3 Low & $5.3561 \mathrm{e}+01$ & $5.3662 \mathrm{e}+01(0.19)$ & $8.8826 \mathrm{e}+01(65.84)$ \\
4 & 4 Low & $7.8934 \mathrm{e}+02$ & $7.9259 \mathrm{e}+02(0.41)$ & $1.5791 \mathrm{e}+02(100.05)$ \\
5 & Shear mode & $1.0165 \mathrm{e}+02$ & $1.0189 \mathrm{e}+02(0.24)$ & $2.4674 \mathrm{e}+02(142.74)$ \\
6 & 5 Low & $1.0448 \mathrm{e}+02$ & $1.0524 \mathrm{e}+02(0.73)$ & $3.5531 \mathrm{e}+02(240.08)$ \\
7 & 1 High & $1.1216 \mathrm{e}+02$ & $1.1251 \mathrm{e}+02(0.31)$ & $4.8361 \mathrm{e}+02(331.18)$ \\
8 & 6 Low & $1.2993 \mathrm{e}+02$ & $1.3140 \mathrm{e}+02(1.13)$ & $6.3165 \mathrm{e}+02(386.15)$ \\
9 & 2 High & $1.3707 \mathrm{e}+02$ & $1.3776 \mathrm{e}+02(0.50)$ & $7.9943 \mathrm{e}+02(483.24)$ \\
10 & 7 Low & $1.5520 \mathrm{e}+02$ & $1.5773 \mathrm{e}+02(1.63)$ & $9.8696 \mathrm{e}+02(535.93)$ \\
\hline
\end{tabular}
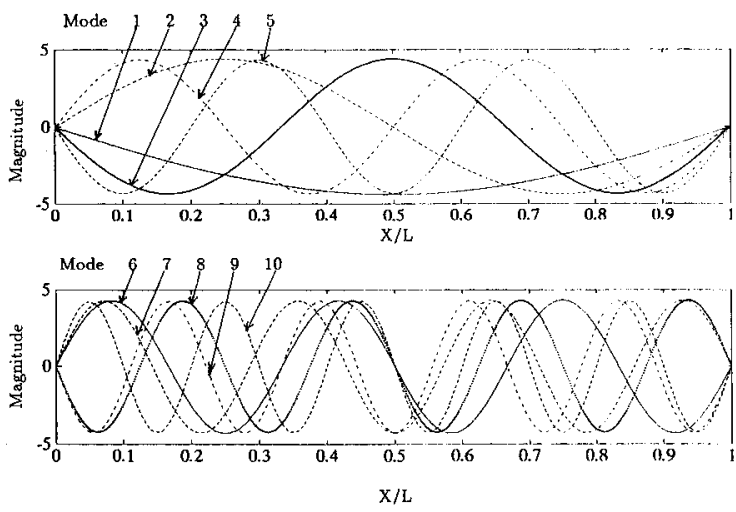

Fig. 1. The first ten mode shape plots for a Timoshenko beam with $v=$ $0.3, \mathrm{k}=0.85$ and $\frac{r}{L}=0.015$.
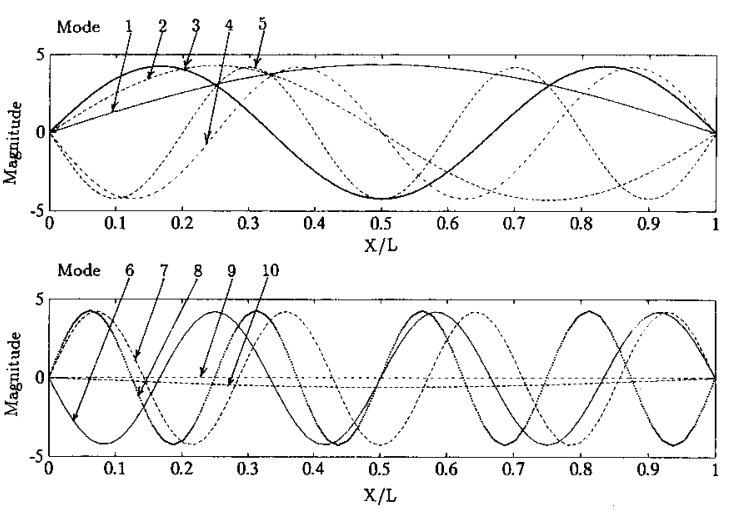

Fig. 2. The first en mode shape plots for a Timoshenko beam with $v=$ $0.3, \mathrm{k}=0.85$, and $\frac{r}{L}=0.045$. 

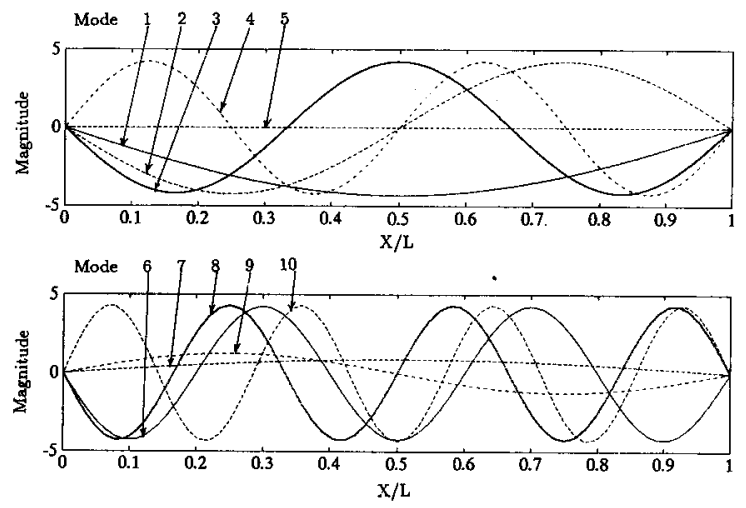

Fig. 3. The first ten mode shape plots for a Timoshenko beam with $v=$ $0.3, \mathrm{k}=0.85$ and $\frac{r}{L}=0.075$.

has only one nodal point. Mode 10, supposed to have nine nodal points in classical analysis, is the seventh mode in the lower spectrum and hence has only six nodal points as is evident in Fig. 3.

It is apparent from the above analysis that the Timoshenko beam model developed here is valid and hence can be used to analyze the moving load problem to be presented in the following section. Note that the model developed in this paper retains the classical one element four degrees of freedom formulation without using additional nodal degrees of freedom as reported in $[23,24]$ which may experience difficulties for analyzing complex structures due to compatibility requirement at the nodes.

\section{MOVING FORCE ON A TIMOSHENKO BEAM}

Fig. 4 shows a beam subjected to a moving concentrated force. When the finite element method is used to solve this class of problems, the governing structural equation can be written as:

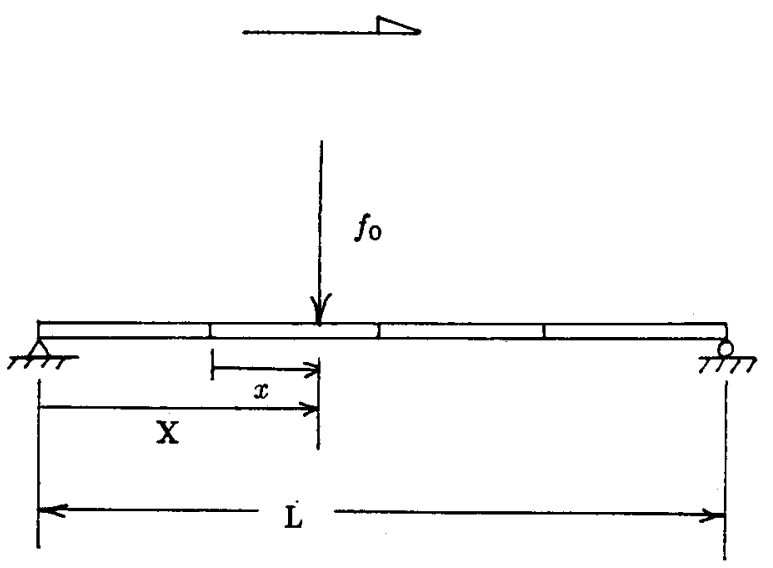

$$
[M]\{\ddot{d}\}+[C]\{\dot{d}\}+[K]\{d\}=\{f\}=[N]^{T} f_{0}
$$

where

[M] : structural mass matrix including rotary inertia effect

$[\mathrm{C}]$ : structural damping matrix

$[\mathrm{K}]$ : structural stiffness matrix including shear effect

$[N]^{T}$ : transposition of the shape functions evaluated at the position of the force, i.e. $x$ in Fig. 4

$f_{0}$ : magnitude of the concentrated force

and $\{d\},\{\dot{d}\}$, and $\{\ddot{d}\}$, denote displacement, velocity, and acceleration vectors respectively. Note that shape functions shown in Eq. (3) should be used for analysis of Timoshenko beams.

Eq. (14) is a system of second order linear differential equations. It should be noted that $[N]^{T}$ is a vector with zero entries except those corresponding to the nodal displacements of the element on which the load is acting. Thus for a beam element with 4 degrees of freedom, the number of non-zero entries within the $n$ by 1 vector will be four, where $n$ is the total number of degrees of freedom of the beam model. This 4 by 1 "sub-vector" is time dependent as the load moves from one position to another within one element. As the load moves to the next element, this sub-vector will shift in position corresponding to the degrees of freedom of the element where the load is positioned. Fig. 5 shows the equivalent nodal loads at the nodal points when a beam element is loaded by a concentrated force, where $f_{L}, M_{L}, f_{R}$, and $M_{R}$ denote the equivalent force and moment at the left and right node of the beam element respectively. The equivalent nodal loads are expressed as a $4 \times 1$ vector and are obtained by multiplying the transpose of the shape functions with the concentrated force, as given on the right hand side of Eq. (14), where the shape functions are evaluated at the position of the given load. When the

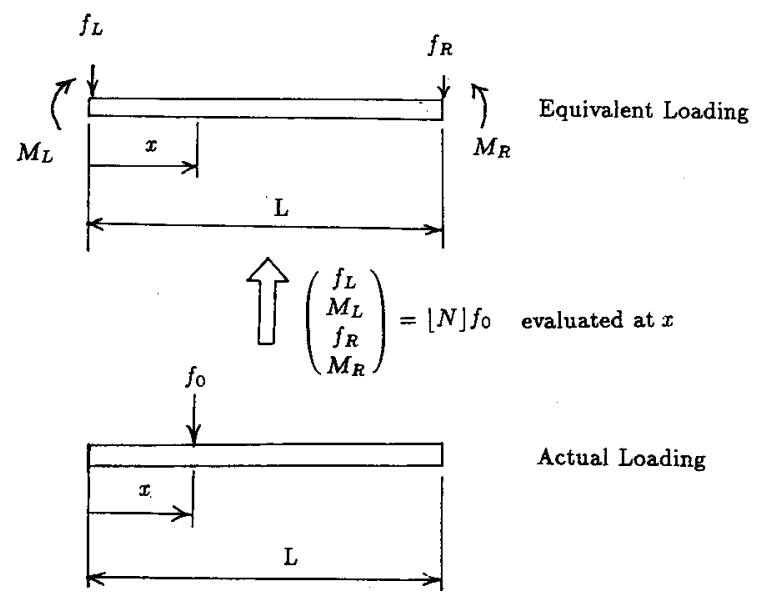

Fig. 4. A beam subjected to a moving concentrated force.

Fig. 5. Equivalent nodal loads for a beam element loaded by a force. 
concentrated force moves to the other position from its current location, the numerical values of the shape functions will change and consequently the equivalent nodal loads will vary accordingly. It should be noted when the concentrated force moves to the other element, the shape functions corresponding to the new element must be used, and this results in a position shift of the equivalent nodal loads within the structural load column vector. Either the modal superposition or the direct step by step integration can be applied to solve the governing equations. However, for this moving force problem, since the system is linear and time invariant, modal superposition will be more efficient than the direct step by step integration and the solution scheme is discussed below.

Eq. (14) can be transformed to modal coordinate so that a set of decoupled equations is obtained. Note that proportional damping is assumed in the present analysis and the mode shapes have been normalized with respect to the structural mass matrix.

$$
\ddot{q}_{i}+2 \xi_{i} w_{n_{i}} \dot{q}_{i}+w_{n_{i}}^{2} q_{i}=f_{i}
$$

for $i=1,2, \ldots, \mathrm{n}$, where $q_{i}, \dot{q}_{i}$, and $\ddot{q}_{i}$ represent the modal displacement, velocity, and acceleration, respectively, $\xi_{i}$ the percent modal damping, $w_{n_{i}}$ undamped circular natural frequency for the $i$-th mode, and $f_{i}$ the modal force obtained from pre-multiplication of the original force vector by the transposition of corresponding modal vector. Since the modal force is time dependent, Duhamel integral can be used to solve for the response and the piecewise-constant solution to Eq. (15) can then be written as:

$$
\begin{aligned}
q_{i, j} & =\frac{F_{i, j}}{w_{n_{i}}^{2}}\left[1-e^{-\xi_{i} w_{n i} \Delta t_{j}}\left\{\cos \left(w_{d_{i}} \cdot \Delta t_{j}\right)\right.\right. \\
& \left.\left.+\frac{\xi_{i} w_{n_{i}}}{w_{d_{i}}} \sin \left(w_{d_{i}} \cdot \Delta t_{j}\right)\right\}\right] \\
& +e^{-\xi_{i} w_{n i} \Delta t_{j}}\left\{q_{i, j-1} \cos \left(w_{d_{i}} \cdot \Delta t_{j}\right)\right. \\
& \left.+\frac{\dot{q}_{i, j-1}+\xi_{i} w_{n_{i}} q_{i, j-1}}{w_{d_{i}}} \sin \left(w_{d_{i}} \cdot \Delta t_{j}\right)\right\}
\end{aligned}
$$

and

$$
\begin{aligned}
& q_{i, j}=\frac{F_{i, j} w_{d_{i}}}{w_{n_{i}}^{2}} e^{-\xi_{i} w_{n i} \Delta t_{j}\left(1+\frac{\xi_{i}^{2} w_{n i}^{2}}{w_{d i}^{2}}\right)} \\
& \sin \left(w_{d_{i}} \cdot \Delta t_{j}\right)+w_{d_{i}} e^{-\xi_{i} w_{n i} \Delta t_{j}} \\
& \times\left\{-q_{i, j-1} \sin \left(w_{d_{i}} \cdot \Delta t_{j}\right)\right.
\end{aligned}
$$

$$
\begin{aligned}
& +\frac{\dot{q}_{i, j-1}+\xi_{i} w_{n_{i}} q_{i, j-1}}{w_{d_{i}}} \cos \left(w_{d_{i}} \cdot \Delta t_{j}\right) \\
& -\frac{\xi_{\mathrm{i}} w_{n_{i}}}{w_{d_{i}}}\left[q_{i, j-1} \cos \left(w_{d_{i}} \cdot \Delta t_{j}\right)\right. \\
& \left.\left.+\frac{\dot{q}_{i, j-1}+\xi_{i} w_{n_{i}} q_{i, j-1}}{w_{d_{i}}} \sin \left(w_{d_{i}} \cdot \Delta t_{j}\right)\right]\right\}
\end{aligned}
$$

where the subscript ' $j$ ' denotes the $j$-th time position in the solution process, $q_{i, j}$ and $\dot{q}_{i, j}$ the $i$-th modal displacement and velocity respectively at the $j$-th time step, $F_{i, j}$ the $i$-th modal force at the $j$-th time step, $w_{d_{i}}$ the damped natural frequency for the $i$-th mode, and $\Delta t_{j}$ the time step. After the moving force leaves the beam span, the beam undergoes free vibration and exact expression is used rather than using Eqs. (16) and (17) which requires the solution from the previous time step. This alleviates possible error accumulation. The analysis results for the above formulation are presented in the following section.

\section{SIMULATION RESULTS}
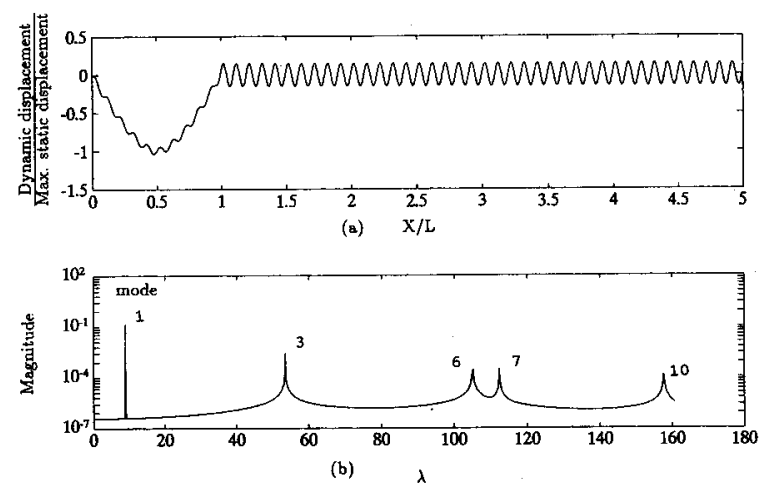

Fig. 6. A simply supported Timoshenko beam subjected to a moving force with $T_{f} \tau=0.1, v=0.3, \mathrm{k}=0.85$, and $\frac{r}{L}=0.075$ (a) dynamic displacement at the beam center (b) Free response FFT for dynamic displacement at the beam center with logrithmic scale.
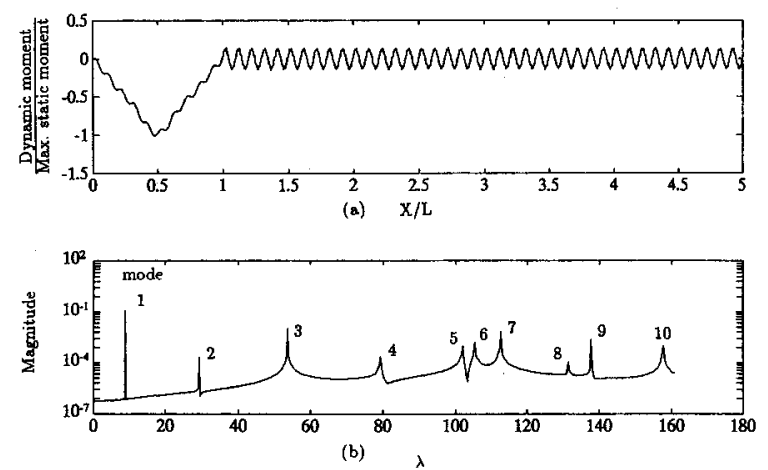

Fig. 7. A simply supported Timoshenko beam subjected to a moving force with $T_{f} / \tau=0.1, v=0.3, \mathrm{k}=0.85$, and $\frac{r}{L}=0.075$ (a) dynamic moment at the beam center (b) Free response FFT for dynamic moment at the beam center with logrithmic scale. 
Figs. 6 and 7 illustrate the dynamic response, moment, and the corresponding modal participation in the free response, respectively, at the beam center with $\mathrm{r} / \mathrm{L}=0.075, T_{f} / \tau=0.1$, where $T_{f}$ is the fundamental period of the support beam and $\tau$ is the time required for the moving force to leave the beam span. The frequency axis is normalized with $\lambda=w_{n} \sqrt{\frac{\rho A L^{4}}{E I}}$. Thirty-two Timoshenko beam elements were used in this analysis. As can be seen from the analysis results, the fundamental mode is undoubtedly the dominant one. In Fig. 6, modes $1,3,6,7$, and 10 are clearly shown with the first mode being the dominant one. The other modes have no contribution for displacement response at the beam center since they are anti-symmetric modes (see Table 3 and Fig. 3). It might be expected that modes 5 and 9 would have contribution to the response at the beam center as in the ordinary analysis for BernoulliEuler's beams. However, in the present study, mode 5 corresponds to the shear mode, which has no transverse displacement, whereas mode 9 is the second high mode, which is an anti-symmetric mode. Although it does not seem to be the same case for the dynamic moment analysis as shown in Fig. 7, with all modes being present. This is simply because the computation of moment requires information from one additional node in addition to the node at the beam center. If more elements are used, the anti-symmetric modes will have little effect.

Figs. 8 and 9 depict the dynamic displacement and moment responses, respectively, for a Timoshenko beam traversed by a moving force with various moving speeds. The static case is also shown for comparison. It can be seen that at lower speed such as $T_{f} \tau=0.1$ and 0.5 , the response oscillates about the static curves. For these two cases, the traveling time $\tau$ is 10 and 2 times, respectively, the fundamental period of the beam and hence the dominant lowest mode has enough time to complete 10 and 2 vibration cycles respectively. The moment response curves appear to be not as smooth as those for

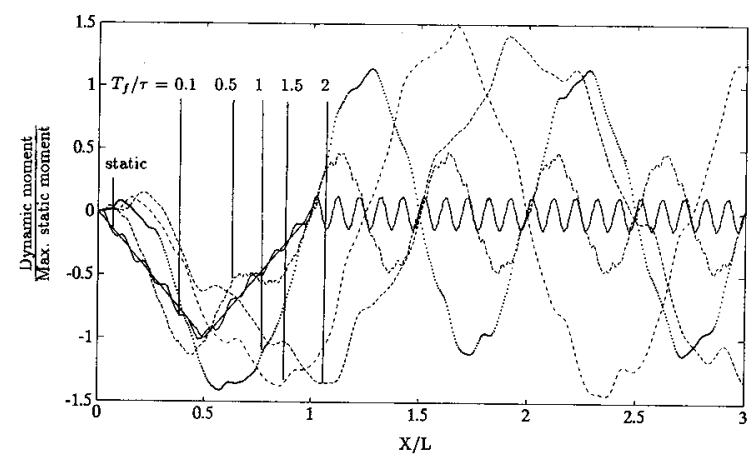

Fig. 8. Dynamic displacement at the beam center for a simply supported Timoshenko beam subjected to a moving force with various moving speeds, $\nu=0.3, \mathrm{k}=0.85$, and $\frac{r}{L}=0.075$.

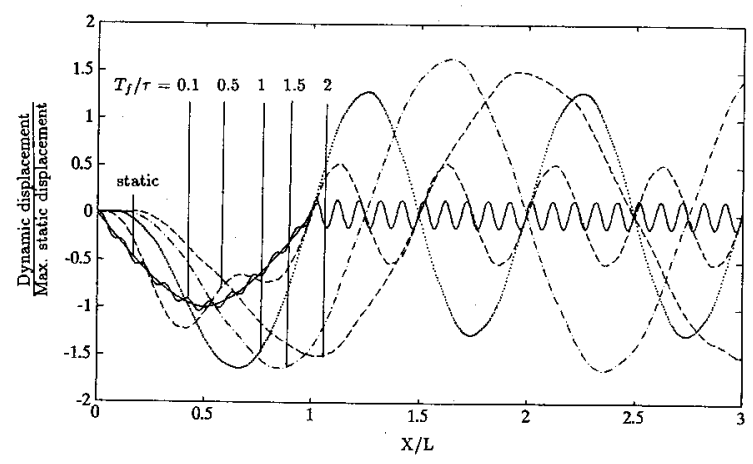

Fig. 9. Dynamic moment at the beam center for a simply supported Timoshenko beam subjected to a moving force with various moving speeds, $v=0.3, \mathrm{k}=0.85$, and $\frac{r}{L}=0.075$.

displacement response. This is because the moment solution converges slower in terms of higher modes since it involves second derivatives of displacement. Dynamic amplification, defined as the ratio between the maximum dynamic response and the maximum static response, with respect to the number of modes being used in the solution process is tabulated in Tables 4 through 6 for three different slenderness ratios with various moving force speeds. The effect of damping, with $\xi_{1}=2 \%$ and $\xi_{2}=5 \%$, is shown in the last column with the first ten modes used for computing response. The mode participation can be clearly seen from these tables. It appears that moment calculation requires more modes for accurate analysis than the displacement computation does. As can be seen from Table 4 through 6 , as far as dynamic displacement is concerned, the use of the fundamental mode yields sufficient accuracy. However, when accurate dynamic stress is required, which calls for precise computation of bending moment, more modes may have to be used depending on the moving load speed and the slenderness ratio, $r / L$. For instance, for $r / L=0.015$ and $T_{f} / \tau=0.1$ the difference for dynamic moment amplification between one mode and ten mode solution is $14.7 \%$, while the difference is only $3 \%$ for $T_{f} / \tau=2$. However, for $\mathrm{r} / \mathrm{L}=$ 0.075 and $T_{f} / \tau=2$ the difference reaches $11.2 \%$. It can also be seen that higher moving speed does not necessarily induce larger dynamic impact to the support beam. For the amount of beam damping given in this study, its effect on reducing the dynamic impact on the support beam due to a moving load is quite limited since the process is of the transient type.

\section{CONCLUSIONS}

This work has examined dynamic finite element analysis for Timoshenko beams subjected to a moving force. It was illustrated that for thick beam structures, the use of Bernoulli-Euler's beam theory yields poor results and the inclusion of shear and rotary inertia effects is crucial for accurate analysis. For the moving force 
Table 4. Dynamic amplification for displacement and moment at the beam center for a simply supported Timoshenko beam subjected to a moving force $v=0.3, \mathrm{k}=0.85$, and $\frac{r}{L}=0.015$.

\begin{tabular}{c|cccccccccccc}
\hline$T_{f} / \tau$ & & & \multicolumn{9}{c}{ No. of modes used } & \multicolumn{3}{c}{$c$} & \multicolumn{3}{c}{$\xi_{1}=2 \%$} \\
& & 1 & 2 & 3 & 4 & 5 & 6 & 7 & 8 & 9 & 10 & $\xi_{2}=5 \%$ \\
\hline \multirow{2}{*}{0.1} & $D_{d}$ & 1.0330 & 1.0330 & 1.0454 & 1.0454 & 1.0470 & 1.0470 & 1.0476 & 1.0476 & 1.0478 & 1.0478 & 1.0258 \\
& $D_{m}$ & 0.8515 & 0.8516 & 0.9398 & 0.9399 & 0.9694 & 0.9696 & 0.9873 & 0.9876 & 0.9985 & 0.9988 & 0.9783 \\
\hline \multirow{2}{*}{1.0} & $D_{d}$ & 1.7045 & 1.7045 & 1.7025 & 1.7025 & 1.7008 & 1.7008 & 1.7003 & 1.7003 & 1.7003 & 1.7003 & 1.6568 \\
& $D_{m}$ & 1.4051 & 1.4049 & 1.4260 & 1.4256 & 1.4226 & 1.4221 & 1.4114 & 1.4111 & 1.4010 & 1.4012 & 1.3491 \\
\hline \multirow{2}{*}{2.0} & $D_{d}$ & 1.5456 & 1.5456 & 1.5502 & 1.5502 & 1.5494 & 1.5494 & 1.5497 & 1.5497 & 1.5496 & 1.5496 & 1.4979 \\
& $D_{m}$ & 1.2741 & 1.2742 & 1.3108 & 1.3105 & 1.3081 & 1.3077 & 1.3107 & 1.3107 & 1.3138 & 1.3138 & 1.2552 \\
\hline
\end{tabular}

Table 5. Dynamic amplification for displacement and moment at the beam center for a simply supported Timoshenko beam subjected to a moving force $v=0.3, \mathrm{k}=0.85$, and $\frac{r}{L}=0.045$.

\begin{tabular}{|c|c|c|c|c|c|c|c|c|c|c|c|c|}
\hline \multirow[t]{2}{*}{$T_{f} / \tau$} & & \multicolumn{10}{|c|}{ No. of modes used } & \multirow{2}{*}{$\begin{array}{l}\xi_{1}=2 \% \\
\xi_{2}=5 \%\end{array}$} \\
\hline & & 1 & 2 & 3 & 4 & 5 & 6 & 7 & 8 & 9 & 10 & \\
\hline \multirow{2}{*}{0.1} & $D_{d}$ & 1.0218 & 1.0218 & 1.0387 & 1.0387 & 1.0419 & 1.0419 & 1.0433 & 1.0433 & 1.0433 & 1.0433 & 1.0218 \\
\hline & $D_{m}$ & 0.8524 & 0.8525 & 0.9446 & 0.9451 & 0.9771 & 0.9828 & 1.0016 & 1.0017 & 1.0017 & 1.0009 & 0.9797 \\
\hline \multirow{2}{*}{1.0} & $D_{d}$ & 1.6860 & 1.6860 & 1.6828 & 1.6828 & 1.6794 & 1.6794 & 1.6871 & 1.6871 & 1.6871 & 1.6871 & 1.6367 \\
\hline & $D_{m}$ & 1.4065 & 1.4051 & 1.4225 & 1.4207 & 1.4187 & 1.4169 & 1.4043 & 1.4036 & 1.4035 & 1.4020 & 1.3595 \\
\hline \multirow{2}{*}{2.0} & $D_{d}$ & 1.5289 & 1.5289 & 1.5354 & 1.5354 & 1.5353 & 1.5353 & 1.5356 & 1.5356 & 1.5356 & 1.5357 & 1.4805 \\
\hline & $D_{m}$ & 1.2754 & 1.2763 & 1.3109 & 1.3122 & 1.3241 & 1.3246 & 1.3298 & 1.3301 & 1.3282 & 1.3280 & 1.2446 \\
\hline
\end{tabular}

Table 6. Dynamic amplification for displacement and moment at the beam center for a simply supported Timoshenko beam subjected to a moving force $v=0.3, \mathrm{k}=0.85$, and $\frac{r}{L}=0.075$.

\begin{tabular}{|c|c|c|c|c|c|c|c|c|c|c|c|c|}
\hline \multirow[t]{2}{*}{$T_{f} / \tau$} & & \multicolumn{10}{|c|}{ No. of modes used } & \multirow{2}{*}{$\begin{array}{l}\xi_{1}=2 \% \\
\xi_{2}=5 \%\end{array}$} \\
\hline & & 1 & 2 & 3 & 4 & 5 & 6 & 7 & 8 & 9 & 10 & \\
\hline \multirow{2}{*}{0.1} & $D_{d}$ & 1.0028 & 1.0028 & 1.0270 & 1.0270 & 1.0270 & 1.0331 & 1.0333 & 1.0333 & 1.0333 & 1.0366 & 1.0126 \\
\hline & $D_{m}$ & 0.8569 & 0.8573 & 0.9563 & 0.9572 & 0.9572 & 0.9949 & 0.9897 & 0.9909 & 0.9904 & 1.0121 & 0.9710 \\
\hline \multirow{2}{*}{1.0} & $D_{d}$ & 1.6547 & 1.6547 & 1.6547 & 1.6547 & 1.6547 & 1.6500 & 1.6502 & 1.6502 & 1.6502 & 1.6066 & 1.6064 \\
\hline & $D_{m}$ & 1.4139 & 1.4106 & 1.4105 & 1.4078 & 1.4078 & 1.4177 & 1.4129 & 1.4102 & 1.4112 & 1.4132 & 1.3767 \\
\hline \multirow{2}{*}{2.0} & $D_{d}$ & 1.5005 & 1.5005 & 1.5134 & 1.5134 & 1.5134 & 1.5190 & 1.5188 & 1.5188 & 1.5188 & 1.5212 & 1.4527 \\
\hline & $D_{m}$ & 1.2821 & 1.2866 & 1.3902 & 1.3908 & 1.3908 & 1.4268 & 1.4258 & 1.4259 & 1.4276 & 1.4438 & 1.2638 \\
\hline
\end{tabular}

problem, modal superposition was used to solve the system equations efficiently. The participation of modes on the structural response was illustrated. Efficient com- putation for dynamic response can be achieved by considering the particular dynamic characteristics of a Timoshenko beam with low and high spectrums being 
present.

\section{ACKNOWLEDGEMENT}

The author is grateful to the National Science Council (NSC), Taiwan, ROC, for the financial support under the contract NSC 81-0403-E-019-514.

\section{LIST OF SYMBOLS}

a: beam element length

A: cross-sectional area of a beam

[C]: structural damping matrix of a beam

$\{d\}$ : structural nodal displacement vector

$\{\dot{d}\}$ : structural nodal velocity vector

$\{\ddot{d}\}$ : structural nodal acceleration vector

$\{d\}_{e}$ : element nodal displacement vector

$\{\dot{d}\}_{e}$ : element nodal velocity vector

$\{\ddot{d}\}_{e}$ : element nodal acceleration vector

$D_{d}$ : dynamic amplification for displacement

$D_{m}$ : dynamic amplification for bending moment

E: Young's modulus of a beam

$f_{0}: \quad$ magnitude of a concentrated force

$F_{i, j}: \quad$ modal force of the $i$-th mode at the $j$-th time step

$f_{L}$ : equivalent force at the left node of a beam element

$f_{R}$ : equivalent force at the right node of a beam element

G: $\quad$ shear modulus

I: $\quad$ area moment of inertia

[K]: structural stiffness matrix of a beam

$\mathrm{k}$ : $\quad$ shear coefficient

$\mathrm{L}$ : length of a beam

$[\mathrm{M}]$ : structural mass matrix of a beam

$M_{L}$ : equivalent moment at the left node of a beam element

$M_{R}$ : equivalent moment at the right node of a beam element

$[N]:$ a row vector denoting shape functions

$[N]^{T}:$ transposition of the shape functions

$[N]_{x}$ : first derivative of the shape functions with respect to $x$

$q_{i}: \quad$ the $i$-th modal displacement

$q_{i, j}: \quad$ the $i$-th modal displacement at the $j$-th time step

$\dot{q}_{i, j}: \quad$ the $i$-th modal velocity at the $j$-th time step

$\ddot{q}_{i}: \quad$ the $i$-th modal acceleration

r: radius of gyration of a beam

$T_{f} \quad$ fundamental period of a support beam

$w$ : vertical displacement of a beam as a function of space and time

$x$ : $\quad$ distance between the contact position of a moving system and the left node of a beam element

$\mathrm{X}$ : distance between the contact position of a moving system and the left end of a support beam

$w_{d_{i}}: \quad$ damped natural frequency of the $i$-th mode $w_{n_{i}}$ : undamped natural frequency of the $i$-th mode

$\xi_{i}$ : percent modal damping of the $i$-th mode of a beam

$\tau$ : $\quad$ time required for a moving system to travel from left end of a beam to the right end

$\rho$ : mass density of a beam

$\gamma$. shear strain

$v$ : Poisson's ratio

\section{REFERENCE}

1. Inglis, C. E. A Mathematical Treatise on Vibration in Railway Bridges, Cambridge University Press (1934).

2. Meirovitch, L., Analytical Methods in Vibrations, The Macmillan Company (1967).

3. Warburton, G.B., The Dynamic Behavior of Structures, Pergamon Press (1976).

4. Fryba, L., Vibration of Solids and Structures under Moving Loads, Nordhoff International Publishing (1972).

5. Katz, R., Lee C.W., Ulsoy, A.G., and Scott, R.A., "Dynamic Stability and Response of a Beam Subjected to a Deflection Dependent Moving Load,' Journal of Vibration, Acoustics, Stress, and Reliability in Design, 109, pp. 361-365 (1987).

6. Wen, R.K., "Dynamic Response of Beams Traversed by Two-axle Loads", ASCE Journal of Engineering Mechanics Division 86 EM 5, pp. 91-111 (1960).

7.Penno, E., "Discussion on Dynamic Response of Beams Traversed by Two-axle Loads,' ASCE Journal of Engineering Mechanics Division, 87 EM 3, 61 (1961).

8. Wen, R.K., "Closure on Dynamic Response of Beams Traversed by Two-axle Loads," ASCE Journal of Engineering Mechanics Division, 87 EM 3, 85 (1961).

9. Hino, J., Yoshimura, T., and Konishi, K., "A Finite Element Method Prediction of the Vibration of a Bridge Subjected to a Moving Vehicle Load,' Journal of Sound and Vibration 96, pp. 45-53 (1984).

10. Hino, J., Yoshimura, T., and Ananthanarayana, N., "Vibration Analysis of Nonlinear Beams Subjected to a Moving Load Using the Finite Element Method," Journal of Sound and Vibration, 100, pp. 477-491 (1985).

11. Yoshimura, T., Hino, J., and Ananthanarayana, N., "Vibration Analysis of Nonlinear Beams Subjected to Moving Loads by Using the Galerkin Method," Journal of Sound and Vibration, 104, pp. 179-186 (1986).

12. Filho, F.V., "Finite Element Analysis of Structures under Moving Loads,' Shock and Vibration Digest, 100, pp. 27-35 (1978).

13.Lin, Y.-H. and Trethewey, M.W., "Finite Element Analysis of Elastic Beams Subjected to Moving Dynamic Loads," Journal of Sound and Vibration, Vol. 136(2), pp. 323-342 (1990).

14.Lin, Y.-H., Trethewey, M.W., Reed, H.M., Shawley, J.D., and Sager, S.J. “Dynamic Modeling and Analysis of a High Speed Precision Drilling Machine," Journal of Vibration and Acoustics, Vol. 112, No. 3, pp. 355- 
365 (1990).

15. Florence, A.L. "Traveling Force on a Timoshenko Beam", Journal of Applied Mechanics, pp. 351-358 (1965).

16. Achenbach, J.D. and Sun, C.T., "Moving Load on a Flexibly Supported Timoshenko Beam", International Journal of Solids and Structures, Vol. 1, pp. 351-358 (1965).

17. Steele, C.R., "The Finite Beam with a Moving Load," Journal of Applied Mechanics, pp. 111-118 (1967).

18. Steele, C.R., "The Timoshenko Beam with a Moving Load," Journal of Applied Mechanics, pp. 481-488 (1968).

19. Narayanaswami, R. and Adelman, H. M., "Inclusion of Transverse Shear Deformation in Finite Element Displacement Formulation," Journal of AIAA, Vol. 12, No. 11, pp. 1613-1614 (1974).

20. Huang, T.C., "The Effect of Rotary Inertia and of
Shear Deformation on the Frequency and Normal Mode Equations of Uniform Beams with Simple End Conditions," Journal of Applied Mechanics, vol. 28, pp. 579-584 (1961).

21. Cowper, G.R., "The Shear Coefficient in Timoshenko's Beam Theory," Journal of Applied Mechanics, vol. 33, pp. 335-340 (1966).

22. Downs, B., "Transverse Vibration of a Uniform Simply Supported Timoshenko Beam without Transverse Displacement," Journal of Applied Mechanics, pp. 671-673 (1976).

23. Kapur, K.K., "Vibrations of a Timoshenko Beam Using a Finite Element Approach," Journal of Acoustical Society of America, vol. 40, pp. 1058-1063 (1966).

24. Nickell, R.E. and Secor, G.A., "Convergence of Consistently Derived Timoshenko Beam Finite Elements," International Journal for Numerical Methods in Engineering, vol. 5, pp. 243-253 (1972). 